\title{
Влияние адсорбции кислорода и фтора на электронную структуру поверхности $\ln \mathrm{Sb}(111)$
}

\author{
(C) А.А. Фукс ${ }^{1}$, А.В. Бакулин ${ }^{1,2,}$, С.Е. Кулькова ${ }^{1,2}$, Н.А. Валишева ${ }^{3}$, А.В. Постников ${ }^{4}$ \\ ${ }^{1}$ Национальный исследовательский Томский государственный университет, \\ 634050 Томск, Россия \\ ${ }^{2}$ Институт фозики прочности и материаловедения Сибирского отделения Российской академии наук, \\ 634055 Томск, Россия \\ ${ }^{3}$ Институт физики полупроводников им. А.В. Ржанова Сибирского отделения Российской академии наук, \\ 630090 Новосибирск, Россия \\ 4 Лотарингский университет, \\ 57078 Мец, Франция \\ ^E-mail: bakulin@ispms.tsc.ru
}

Поступила в Редакцию 14 января 2019 г.

В окончательной редакции 3 апреля 2019 г.

Принята к публикации 13 мая 2019 г.

\begin{abstract}
Методом проекционных присоединенных волн изучена энергетика связи кислорода и фтора на поверхности $\operatorname{InSb}(111)$ в зависимости от ее окончания. Показано, что на поверхности с индиевым окончанием адсорбция фтора в зависимости от его концентрации приводит к частичному или полному удалению из запрещенной щели поверхностных состояний, индуцированных адсорбцией кислорода. Проникновение обоих адсорбатов в подповерхностные слои приводит к разрушению In-Sb-связей и формированию химических связей фтора и кислорода с приповерхностными атомами подложки, что является начальным этапом образования фторсодержащего анодного оксидного слоя. В случае поверхности $\operatorname{InSb}(111)$, оканчивающейся сурьмой, адсорбция кислорода способствует понижению плотности поверхностных состояний в запрещенной щели. Обсуждаются общие тенденции в изменении электронной структуры поверхности (111) при коадсорбции фтора и кислорода в ряду $\mathrm{A}^{\mathrm{III}} \mathrm{B}^{\mathrm{V}}$ полупроводников.
\end{abstract}

Ключевые слова: $\mathrm{A}^{\mathrm{III}} \mathrm{B}^{\mathrm{V}}$, поверхность (111), адсорбция, электронная структура.

DOI: $10.21883 /$ FTP.2020.01.48760.9063

\section{1. Введение}

Свойства поверхностей бинарных полупроводниковых соединений $\mathrm{A}^{\mathrm{III}} \mathrm{B}^{\mathrm{V}}$ зависят от их ориентации, окончания, реконструкции, а также имеющихся дефектов [1]. Изучение атомной и электронной структуры поверхностей полупроводников $\mathrm{A}^{\mathrm{III}} \mathrm{B}^{\mathrm{V}}$ принципиально важно для разработки новых материалов на основе последних для технологических приложений в микроэлектронике, а также для совершенствования современных нанотехнологий [2-5]. Известно, что для производства нового поколения приборов и устройств на основе гетероструктур металл-диэлектрик-полупроводник (МДП) формирование границ раздела полупроводник-оксид с низкой плотностью интерфейсных состояний является ключевой проблемой [6].

В настоящее время существуют различные способы модификации поверхности полупроводника, которые позволяют существенно понизить плотность поверхностных состояний (ППС) [6,7], в том числе, изменением химического состава окислительной среды. В [8] было показано, что выращивание на поверхности $\operatorname{InAs}(111) \mathrm{A}$ с индиевым окончанием тонкого (10-15 нм) фторсодержащего анодного оксидного слоя позволяет получить на границе раздела InAs/оксид ППС $<5 \cdot 10^{10} \mathrm{~cm}^{-2} \cdot{ }^{-1}$. В то же время при пассива- ции поверхности InAs в электролите без фтора ППС оказывается на 2 порядка выше $\left(2 \cdot 10^{12} \mathrm{~cm}^{-2} \cdot{ }^{-1}\right)$. Значительное снижение ППС наблюдается также при фторировании поверхности $\operatorname{GaAs}(001)$ окислением в плазме $\mathrm{N}_{2} \mathrm{O}-\mathrm{CF}_{4}$ [9]. Исследование фазового состава формирующихся оксидных слоев на InAs и GaAs методом рентгеновской фотоэлектронной спектроскопии (РФЭС) показало, что они содержат фториды элементов III группы и оксифториды мышьяка $[10,11]$. По мнению авторов работы [10], образование последних и приводит к устранению поверхностных состояний из запрещенной щели. Таким образом, формирование промежуточного фторсодержащего анодного оксидного слоя (ФАОС) является необходимым фактором для получения МДП структур с низкой плотностью состояний на границе раздела полупроводник-оксид. Известно, что структуры $\mathrm{Au} /$ ФАОC/InAs(111) и $\operatorname{In}_{2} \mathrm{O}_{3} / \mathrm{SiO}_{2} /$ ФАОC/InAs(111) демонстрируют плотность интерфейсных состояний $<5 \cdot 10^{10}$ эВ $^{-1} \cdot \mathrm{cm}^{-2}[12-14]$, тогда как в случае GaAs возможно понижение ПС на границе раздела ФAOC/GaAs в 50 раз (до $<5 \cdot 10^{11}$ эВ $^{-1} \cdot \mathrm{cm}^{-2}$ ) по сравнению с интерфейсом, полученным без фтора [15].

Сложный состав переходной области между анодным оксидным слоем (АОС), состоящим из смеси оксидов элементов полупроводника разного состава, и полупроводником (AOC/In $(\mathrm{Ga}) \mathrm{As})$ препятствует установлению 
природы состояний в запрещенной щели экспериментальными методами и требует привлечения теоретических методов. В настоящее время имеются единичные работы [16-18], в которых методами из первых принципов в рамках теории функционала электронной плотности (ФЭП) изучалась адсорбция кислорода или фтора на $\mathrm{A}^{\mathrm{III}} \mathrm{B}^{\mathrm{V}}$ поверхностях. Например, в работе [16] $X \alpha$-методом изучалась адсорбция кислорода на обоих окончаниях нереконструированной поверхности $\mathrm{GaAs}(001)-(1 \times 1)$. Было установлено, что понижение ППС наблюдается только на поверхности, оканчивающейся галлием, тогда как кислород практически не влияет на поверхностные состояния в случае окончания поверхности мышьяком. В [17] методом линейных комбинаций атомных орбиталей изучалась адсорбция молекулярного кислорода на Аs-стабилизированной поверхности $\operatorname{GaAs}(001)-(2 \times 4)$ с $\beta 2$-реконструкцией. В этом случае кислород диссоциирует на поверхности, и при образовании $\mathrm{Ga}-\mathrm{O}-\mathrm{As}$ конфигурации состояния в запрещенной щели отсутствуют. Напротив, индуцированные кислородом состояния присутствуют в щели в случае образования его связей с димеризоваными атомами мышьяка. В работе [17] был сделан вывод, что поверхностные состояния являются результатом нарушения структуры вследствие внедрения кислорода в приповерхностные слои. В [18] методом псевдопотенциала изучалась адсорбция атомарного кислорода также на поверхности $\mathrm{GaAs}(001)$ с $\beta 2(2 \times 4)$-реконструкцией. Было показано, что насыщение так называемых „задних“ связей вследствие адсорбции одного или двух атомов кислорода не ведет к появлению дефектных состояний в запрещенной щели, тогда как разрыв димерных связей атомов мышьяка и их взаимодействие с кислородом ведет к пиннингу уровня Ферми $\left(E_{\mathrm{F}}\right)$.

Несколько слов необходимо сказать об изучении адсорбции галогенов на $\mathrm{A}^{\mathrm{III}} \mathrm{B}^{\mathrm{V}}$ поверхностях. Так, адсорбция хлора изучалась на поверхности $\operatorname{GaAs}(001)$ в [19]; при этом было установлено, что хлор предпочитает образовывать связи как с димерными, так и недимерными атомами галлия. Данный вывод был подтвержден в наших ранних работах [20-26] при изучении адсорбции галогенов на (001)- и (111)-поверхностях полупроводников GaAs и InAs. Было установлено, что независимо от реконструкции $\mathrm{A}^{\mathrm{III}} \mathrm{B}^{\mathrm{V}}$ поверхности галогены предпочитают образовывать связи с катионами. Отметим, что в указанных работах взаимодействие электроотрицательных адсорбатов с полупроводниковыми подложками $\mathrm{A}^{\mathrm{III}} \mathrm{B}^{\mathrm{V}}$ изучалось первопринципными методами в рамках ФЭП. В работе [21] также изучалась энергетика связи кислорода на нереконструированной $(1 \times 1)$ и реконструированной $(2 \times 2)$ поверхности $\operatorname{InAs}(111) \mathrm{A}$. Было показано, что адсорбция атомарного кислорода во всех изученных позициях приводит к появлению поверхностных состояний в запрещенной зоне, однако с повышением концентрации кислорода на реконструированной поверхности дефектные состояния локализуются лишь на границе зоны Бриллюэна и смещаются к дну зоны проводимости (ЗП) или к вершине валентной зоны (В3). При адсорбции фтора на нереконструированной поверхности $\operatorname{InAs}(111) A$, как и при ее реконструкции, поверхностные состояния полностью устраняются. Кроме того, было установлено, что наведенные адсорбцией кислорода состояния в запрещенной щели могут быть полностью или частично убраны фтором, когда он образует связи с поверхностными атомами мышьяка.

Таким образом, проведенные нами расчеты указывают на общность механизмов понижения плотности поверхностных состояний в запрещенной щели в случае арсенидов галлия и индия [21,25]. В то же время остается неясным,будет ли замена аниона приводить к изменению особенностей влияния фтора и кислорода на электронную структуру $\mathrm{A}^{\mathrm{III}} \mathrm{B}^{\mathrm{V}}$ поверхности. Другими словами, существуют ли общие тенденции в изменении поверхностной электронной структуры полупроводников $\mathrm{A}^{\mathrm{III}} \mathrm{B}^{\mathrm{V}}$ при коадсорбции фтора и кислорода?

В этой связи цель настоящей работы состоит в изучении электронных свойств поверхности $\operatorname{InSb}(111)$ при адсорбции кислорода и фтора, а также при их коадсорбции, чтобы вскрыть влияние аниона на механизмы пассивации поверхности в зависимости от ее окончания.

\section{2. Метод расчета}

Атомная и электронная структура поверхности $\operatorname{InSb}(111)$ рассчитывалась методом проекционных присоединенных волн (PAW) в плосковолновом базиce $[27,28]$, реализованным программным комплексом VASP $[29,30]$. Использовалось обобщенное градиентное приближение (GGA-PBE) [31] для обменно-корреляционного функционала. Теоретический параметр решетки InSb, равный $6.651 \AA$, на $2.65 \%$ превышает экспериментальное значение $6.479 \AA$ [32]. Структура InSb в направлении [111] представляет собой чередование атомных слоев индия и сурьмы, поэтому поверхность $\operatorname{InSb}(111)-(1 \times 1)$, в зависимости от ее окончания, представлялась восьми- или девятислойными пленками, разделенными промежутками вакуума $\sim 10 \AA \AA$. Оборванные связи сурьмы на одной стороне пленок насыщались атомами псевдоводорода (по одному атому $\mathrm{H}^{*}$ на один атом $\mathrm{Sb}$ ) с дробным зарядом 0.75e [33]. Длина связи $\mathrm{Sb}-\mathrm{H}^{*}$ определялась минимизацией полной энергии системы и была равна $1.761 \AA$. Таким образом, эта сторона пленки имела объемоподобный вид. Позиции атомов псевдоводорода, а также атомов двух ближайших слоев фиксировались, тогда как положения атомов других слоев релаксировались до достижения сил на атомах $\sim 0.01$ эВ/А. Энергия обрезания плоских волн из базисного набора составляла 500 эВ. При интегрировании по зоне Бриллюэна использовалась сетка $k$-векторов $7 \times 7 \times 1$, полученная по схеме Монхорста-Пака [34].

Энергия связи кислорода/фтора с подложкой рассчитывалась по формуле

$$
E_{b}=-[E((\mathrm{O} / \mathrm{F}) / \mathrm{InSb})-E(\mathrm{InSb})-E(\mathrm{O} / \mathrm{F})],
$$

где $E((\mathrm{O} / \mathrm{F}) / \mathrm{InSb})$ - полная энергия системы с адсорбированным атомом кислорода или фтора, $E(\mathrm{InSb})-$ 


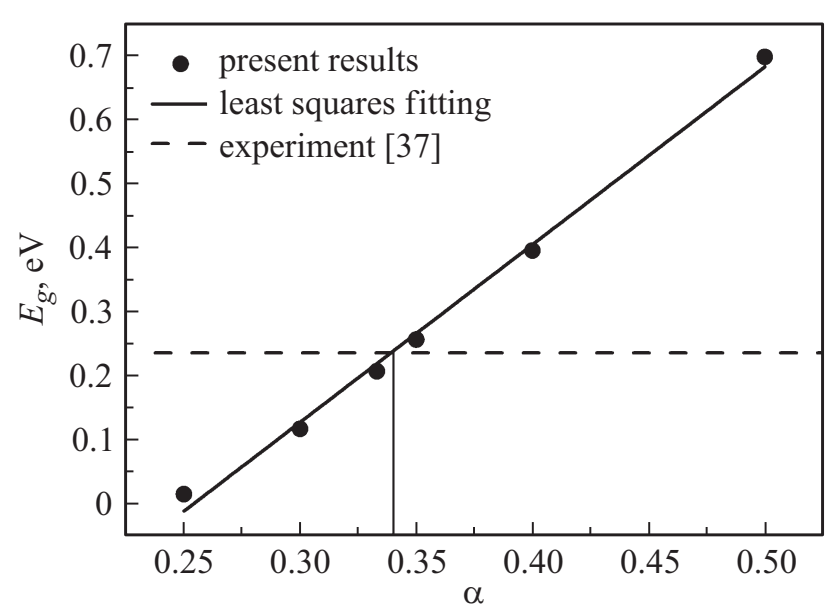

Рис. 1. Зависимость ширины запрещенной щели от параметpa $\alpha$.

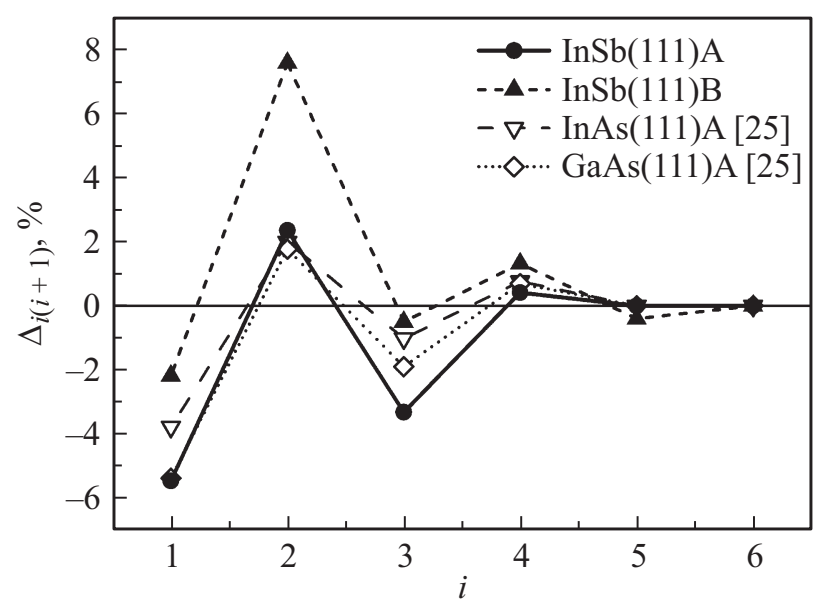

Рис. 2. Релаксация межслоевых расстояний для двух окончаний поверхности $\mathrm{InSb}(111)$ в сопоставлении с данными для катионного окончания $\operatorname{InAs}(111)$ и $\mathrm{GaAs}(111)$.

полная энергия поверхности без адсорбата, $E(\mathrm{O} / \mathrm{F})-$ энергия атомов кислорода/фтора.

Для расчетов электронных энергетических спектров использовался метод гибридных функционалов $[35,36]$, в рамках которого обменно-корреляционный член функционала электронной плотности представляется в виде

$$
\begin{aligned}
E_{x c}^{\mathrm{XSE}}= & \alpha E_{x}^{\mathrm{HF}, \mathrm{sr}}(\mu)+(1-\alpha) E_{x}^{\mathrm{GGA}, \mathrm{sr}}(\mu) \\
& +E_{x}^{\mathrm{GGA}, \operatorname{lr}}(\mu)+E_{c}^{\mathrm{GGA}}(\mu),
\end{aligned}
$$

где $E_{x}^{\mathrm{HF}, \mathrm{sr}}(\mu)$ и $E_{x}^{\mathrm{GGA}, \mathrm{sr}}(\mu)-$ короткодействующие части обменного члена в приближении Хартри-Фока и стандартного обобщенного градиентного приближения, $E_{x}^{\mathrm{GGA}}, \operatorname{lr}(\mu)$ - дальнодействующая часть обменного члена в GGA-приближении, $E_{c}^{\mathrm{GGA}}(\mu)-$ стандартный корреляционный член в GGA-приближении. Размерный параметр $\mu$ отвечает за разделение обменного члена на короткодействующий и дальнодействующий. В рамках HSE06 подхода, использованного в настоящей работе, этот параметр полагался равным $0.2 \AA^{-1}$. Другой параметр, $\alpha$, определяет долю обменной энергии, рассчитанной в приближении Хартри-Фока, в короткодействующей части обменного функционала. Тестовые расчеты показали, что при $\alpha=0.34$ (рис. 1) достигается наилучшее согласие ширины запрещенной щели с экспериментальным значением 0.235 эВ [37].

\section{3. Результаты и их обсуждение}

\section{1. Атомная и электронная структура поверхности $\operatorname{lnSb}(111)$}

На рис. 2 приведены релаксации межслоевых расстояний, которые рассчитывались по следующей формуле:

$$
\Delta_{i j}=\left(d_{i j}-d\right) / d,
$$

где $d-$ расстояние между атомными плоскостями в объеме, $d_{i j}$ - расстояние между атомными слоями $i$ и $j$. Видно, что независимо от окончания $\operatorname{InSb}(111)$ поверхности релаксация носит знакопеременный характер и затухает вглубь пленки. Аналогичная ситуация наблюдалась и на поверхностях $\operatorname{InAs}(111)$ и $\mathrm{GaAs}(111)$ с катионным окончанием $[21,25]$. В случае окончания поверхности атомным слоем аниона сжатие первого и третьего межслоевого расстояния является наименьшим по модулю, по сравнению с катионными поверхностями, а растяжение второго и четвертого расстояния принимает наибольшие значения.

Из рис. 3, $a$ видно, что в случае $\operatorname{InSb}(111)$ А имеется поверхностное состояние в запрещенной зоне (кривая 1), которое фиксирует уровень Ферми. Это состояние распространяется практически по всей двумерной зоне Бриллюэна (ЗБ), при этом основной вклад в него дают $p_{z}$-орбитали поверхностного индия. Аналогичная картина наблюдалась на спектре поверхности (111)А полупроводников InAs и GaAs [21,25]. Отметим, что проекции объемных состояний на рис. 3 показаны штриховкой, а черными и белыми точками обозначены поверхностные состояния индия и сурьмы соответственно. Размер точек пропорционален степени локализации электронных состояний на поверхностных атомах. Совмещение спектра и проекции объемных состояний проводилось по низколежащим $s$-Sb состояниям. В случае окончания поверхности атомным слоем сурьмы поверхностные состояния в большей степени локализованы вблизи точек $\bar{M}$ и $\bar{K}$ двумерной ЗБ и образованы преимущественно состояниями сурьмы (кривые 1 и 2 на рис. $3, b$ ). Следующее поверхностное состояние (кривая 3), локализованное в точке $\bar{K}$ при энергиях $\sim-1.35$ эВ, является смешанным.

\section{2. Адсорбция кислорода и фтора}

Адсорбция кислорода и фтора рассматривалась в вершинных $(T)$ позициях над атомами In или $\mathrm{Sb}$ по- 

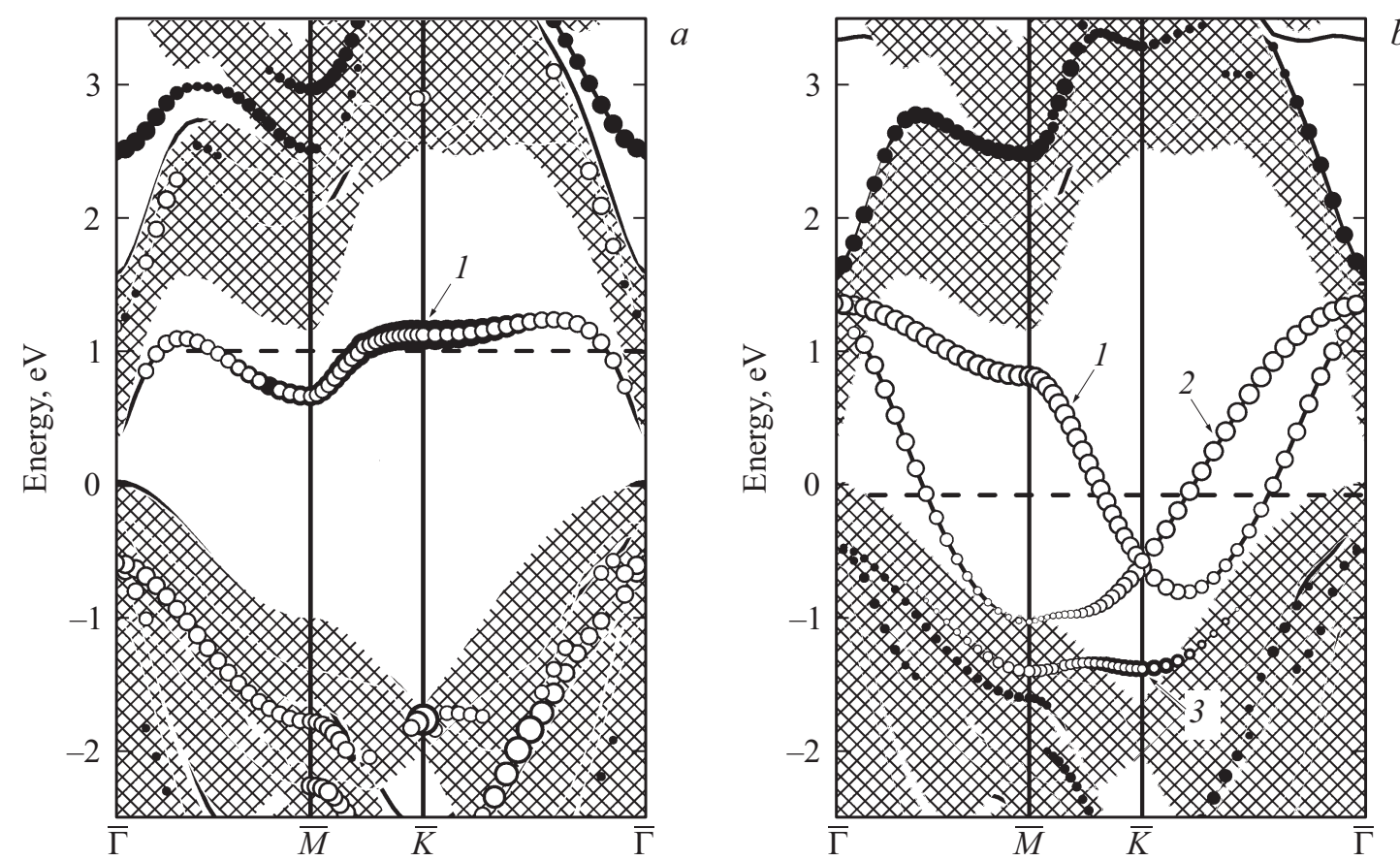

Рис. 3. Электронный энергетический спектр $\operatorname{InSb}(111)-(1 \times 1)$ с In- $(a)$ и $\mathrm{Sb}$-окончанием $(b)$. Здесь и далее горизонтальной пунктирной линией отмечено положение уровня Ферми в пленке.

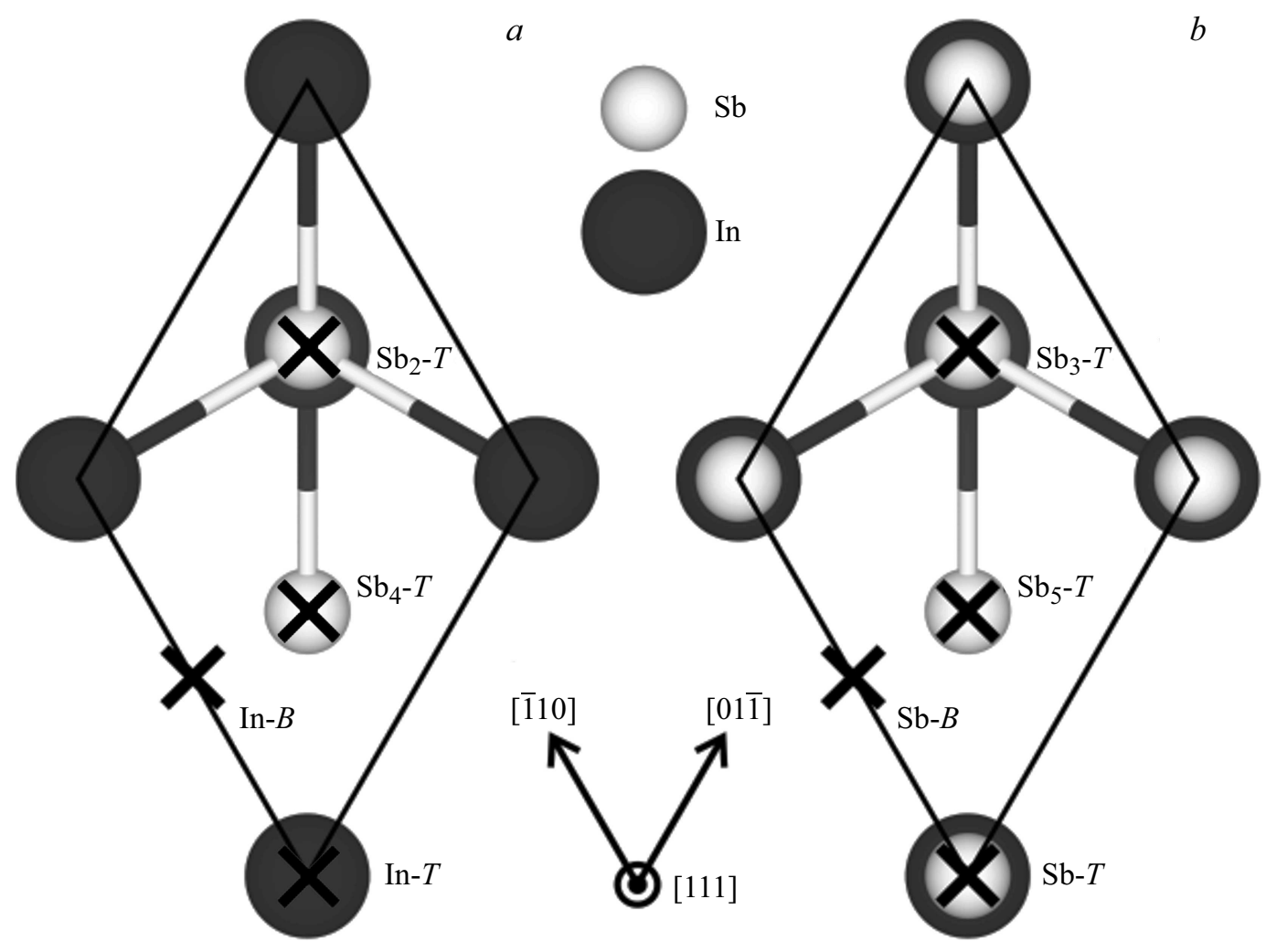

Рис. 4. Позиции адсорбции кислорода и фтора на поверхности $\operatorname{InSb}(111) \mathrm{A}(a)$ и $\operatorname{InSb}(111) \mathrm{B}(b)$.

верхностного слоя, мостиковых $(B)$ позициях - между двумя атомами подложки, а также над атомами сурьмы второго $\left(\mathrm{Sb}_{2}-\mathrm{T}\right)$ и четвертого $\left(\mathrm{Sb}_{4}-\mathrm{T}\right)$ от поверхности слоев в случае In-окончания поверхности или над атомами сурьмы третьего $\left(\mathrm{Sb}_{3}-T\right)$ и пятого $\left(\mathrm{Sb}_{5}-\mathrm{T}\right)$ слоев в случае $\mathrm{Sb}$-окончания (рис. 4). Энергии связи адсорбированных атомов фтора и кислорода на поверхности $\operatorname{InSb}(111)-(1 \times 1)$ представлены в 
Энергия связи фтора и кислорода (в эВ) на поверхности $\operatorname{InSb}(111)$ А и кислорода на $\operatorname{InSb}(111) \mathrm{B}$

\begin{tabular}{c|c|c|c|c}
\hline Позиция & $\mathrm{A}-B$ & $\mathrm{~A}-T$ & $\mathrm{~B}_{2}-T$ & $\mathrm{~B}_{4}-T$ \\
\hline$E_{b}$, эВ & \multicolumn{4}{|c}{ Фтор } \\
\hline $\mathrm{InSb}(111)_{\mathrm{In}}$ & 4.54 & 5.14 & 4.22 & 4.42 \\
$\mathrm{InAs}(111)_{\mathrm{In}}[25]$ & 4.94 & 5.31 & 4.21 & 4.87 \\
$\mathrm{GaAs}(111)_{\mathrm{Ga}}[25]$ & 4.64 & 5.67 & 3.94 & 4.56 \\
\hline$E_{b}$, эB & \multicolumn{5}{|c}{ Кислород } \\
\hline $\mathrm{InSb}(111)_{\mathrm{In}}$ & 5.93 & 4.35 & 4.98 & 4.84 \\
$\mathrm{InAs}(11)_{\mathrm{In}}[25]$ & 6.13 & 4.50 & 5.06 & 5.05 \\
$\mathrm{GaAs}(111)_{\mathrm{Ga}}[25]$ & 5.90 & 5.07 & 4.61 & 4.83 \\
\hline Позиция & В-B & В-T & В $_{3}-T$ & $\mathrm{~B}_{5}-T$ \\
\hline $\mathrm{InSb}(111)_{\mathrm{Sb}}$ & 6.05 & 5.30 & 5.78 & 5.81
\end{tabular}

таблице. Видно, что фтор на поверхности $\operatorname{InSb}(111) \mathrm{A}$ имеет наибольшую энергию связи в вершинной позиции над атомами катиона, тогда как атомы кислорода предпочитают адсорбироваться в мостиковой позиции. Предпочтительность мостиковой позиции связана с тем фактом, что кислород связывается с двумя атомами индия поверхностного слоя (длина связи $2.37 \AA$ ) и атомом сурьмы подповерхностного слоя (длина связи $2.06 \AA$ ), к которому он смещается в процессе релаксации. Энергии связи кислорода с подложкой практически одинаковы в случае адсорбции над атомами сурьмы второго и четвертого от поверхности слоев. Необходимо отметить, что при адсорбции кислорода в $\mathrm{Sb}_{2}$-позиции атом кислорода располагается практически в поверхностном слое (лишь на $0.06 \AA$ ниже поверхности) и образует связь с подповерхностным $\mathrm{Sb}$-атомом (длина связи $-1.97 \AA$ ), тогда как в $\mathrm{Sb}_{4}$-позиции адатом значительно смещается в сторону подложки и находится на $0.88 \AA$ ниже поверхности. Напомним, что в случае $\operatorname{InAs}(111) \mathrm{A}$ и $\operatorname{GaAs}(111) \mathrm{A}$ кислород находился на 0.24 и $0.34 \AA$ выше поверхности соответственно. Проникновение кислорода в подповерхностную область обусловлено бо́льшим параметром решетки InSb по сравнению с указанными выше полупроводниками.

Отмеченные особенности в энергетике связи кислорода с поверхностью имеют место и на $\operatorname{InSb}(111) \mathrm{B}$, оканчивающейся сурьмой (см. таблицу). Атом кислорода предпочитает также адсорбироваться в мостиковой позиции (длина связи $\mathrm{O}-\mathrm{Sb}-2.35 \AA$ ), при этом смещение в сторону подповерхностных In-атомов не наблюдается, что может быть обусловлено большим межслоевым расстоянием $d_{12}$. Заметим, что энергии связи кислорода при адсорбции над атомами сурьмы третьего или пятого слоев также отличаются незначительно.

Из таблицы видно, что энергия связи кислорода в мостиковой позиции на поверхности $\operatorname{InSb}(111)$ А незначительно (на $\sim 0.1$ эВ) отличается от $E_{b}$ в соответству- ющей позиции на $\operatorname{InSb}(111)$ B. В то же время разница в энергиях связи кислорода в других позициях на разных окончаниях поверхности $\operatorname{InSb}(111)$ достигает $\sim 1$ эВ. Энергия связи кислорода на поверхности $\operatorname{InSb}(111)$ на $\sim 0.8-0.9$ эВ больше, чем энергия связи фтора.

Адсорбция кислорода на поверхности $\operatorname{InSb}(111) \mathrm{A}$ приводит к появлению дополнительных поверхностных состояний в запрещенной щели, при этом их структура сильно зависит от положения адсорбата на поверхности (рис. $5, a-d)$. Поверхностные состояния возникают главным образом из-за сильной гибридизации $s, p$-орбиталей кислорода с $s, p$-состояниями сурьмы подповерхностного слоя. Из рис. 5, $a$ видно, что поверхностные состояния, индуцированные O-In-гибридизацией, распространяются практически по всей двумерной ЗБ в случае адсорбции кислорода в In-B позиции; при этом состояния, локализованные вблизи $\bar{\Gamma}$-точки, в основном обусловлены атомами сурьмы подповерхностного слоя. Интересно, что при адсорбции кислорода в In- $T$ позиции в запрещенной щели находятся в основном состояния адатома кислорода и подповерхностного атома сурьмы. Поскольку в In $-T$ позиции кислород связывается только c In поверхностного слоя (длина связи равна $1.99 \AA)$, то $\mathrm{O}-\mathrm{Sb}$ взаимодействие является не прямым (расстояние между O- и $\mathrm{Sb}$-атомами составляет $4.15 \AA$ ), а осуществляется посредством гибридизации с орбиталями индия. Анализ зарядового переноса по методу Бадера (Bader charge transfer) [38] показал, что в этом случае имеет место не только уход заряда с In-атома $(0.46 e)$, но и с атома сурьмы второго слоя $(0.13 e)$. Отметим, что зарядовые состояния атомов более глубоких слоев практически не изменяются. В результате опустошения состояний атомов двух поверхностных слоев в запрещенную щель выходят Sb состояния, a In зона, которая обусловливала пиннинг уровня Ферми на чистой поверхности (кривая 1 на рис. $3, a$ ), полностью смещается в зону проводимости (рис. $5, b$ ). При адсорбции в $\mathrm{Sb}_{2}-T$ позиции атом кислорода непосредственно связывается с подповерхностным атомом сурьмы, что приводит к сильному опустошению состояний сурьмы и к переносу заряда $\sim 0.70 e$ к кислороду, тогда как от индия перенос заряда составляет лишь $0.29 e$. Это приводит также к значительному смещению Sb-состояний в сторону зоны проводимости (кривые 1 и 2 на рис. 5,c). $\mathrm{B} \mathrm{Sb}_{4}-T$ позиции $\mathrm{O}$ адатом находится практически в подповерхностном $\mathrm{Sb}$-слое, при этом межатомное $\mathrm{O}-\mathrm{Sb}$ расстояние составляет $2.72 \AA$, что значительно превышает расстояние между этими атомами в предыдущем случае $(1.97 \AA)$. В результате перенос заряда от атомов $\operatorname{In}_{1}$ и $\mathrm{Sb}_{2}$ к кислороду оказывается несколько меньше, что и отражается на меньшем смещении поверхностных состояний сурьмы и индия в сторону СВ и объясняет наличие большой плотности кислородных состояний выше уровня Ферми (кривые $1-3$ на рис. $5, d)$.

Адсорбция кислорода на поверхности $\mathrm{InSb}(111)$ В также приводит к существенным изменениям ее электрон- 

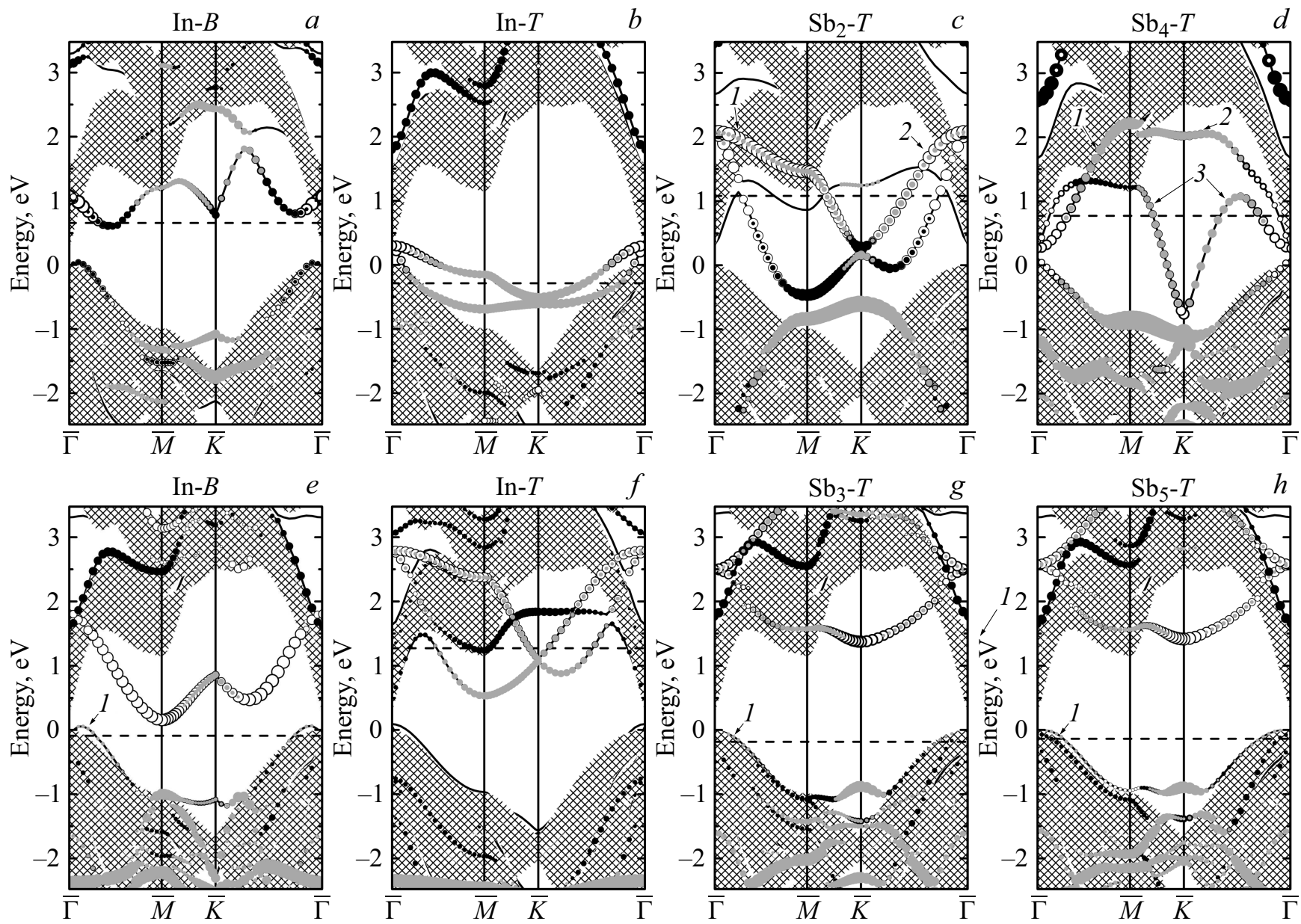

Рис. 5. Электронные энергетические спектры поверхности $\operatorname{InSb}(111)$ с $\operatorname{In}(a-d)$ и $\mathrm{Sb}(e-h)$ окончанием при адсорбции кислорода.
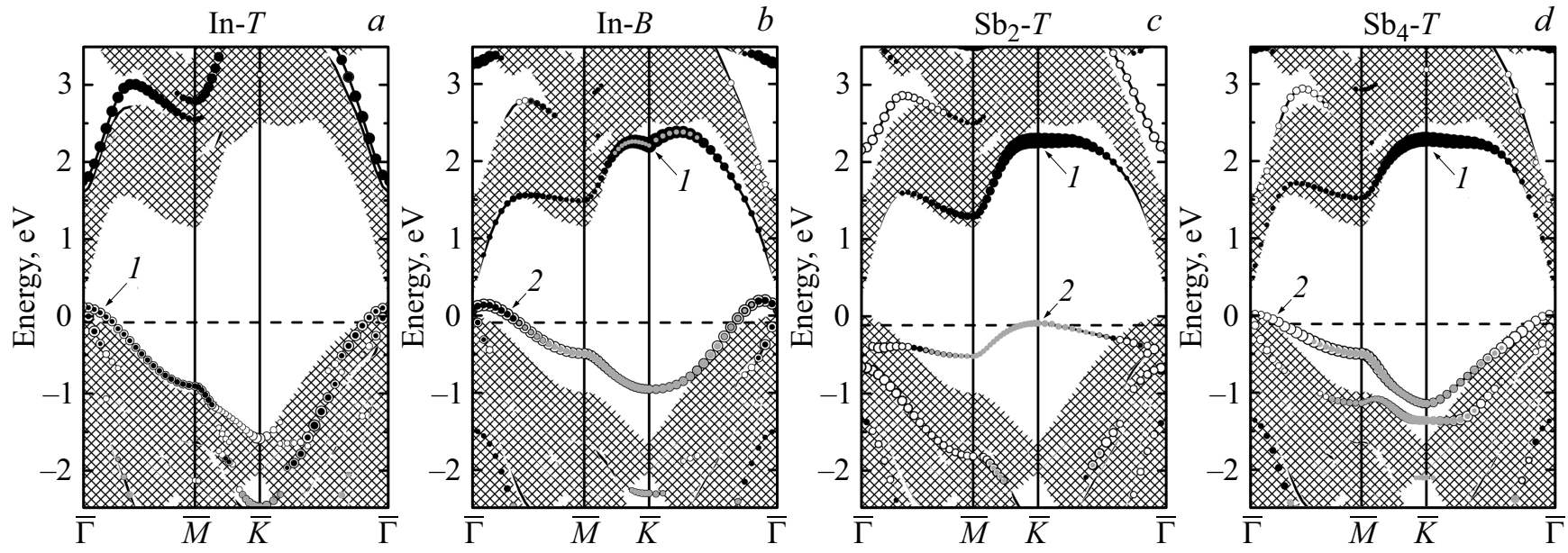

Рис. 6. Электронные энергетические спектры поверхности $\operatorname{InSb}(111) \mathrm{A}$ при адсорбции фтора.

ной структуры (рис. 5,e-h), однако во всех позициях, за исключением наименее предпочтительной - вершинной, кислород уменьшает плотность состояний в запрещенной щели. Те состояния, которые находятся на уровне Ферми (кривая 1 на рис. $5, e, g, h)$, обусловлены главным образом не поверхностными $\mathrm{Sb}_{1}$ или
$\mathrm{In}_{2}$ атомами, а атомами более глубоких слоев. Таким образом, на $\mathrm{Sb}$-окончании поверхности $\operatorname{InSb}(111)$ адсорбция кислорода приводит к понижению плотности поверхностных состояний. Данное заключение согласуется с экспериментальными данными по окислению поверхности $\mathrm{InSb}(111)$ [39]. 

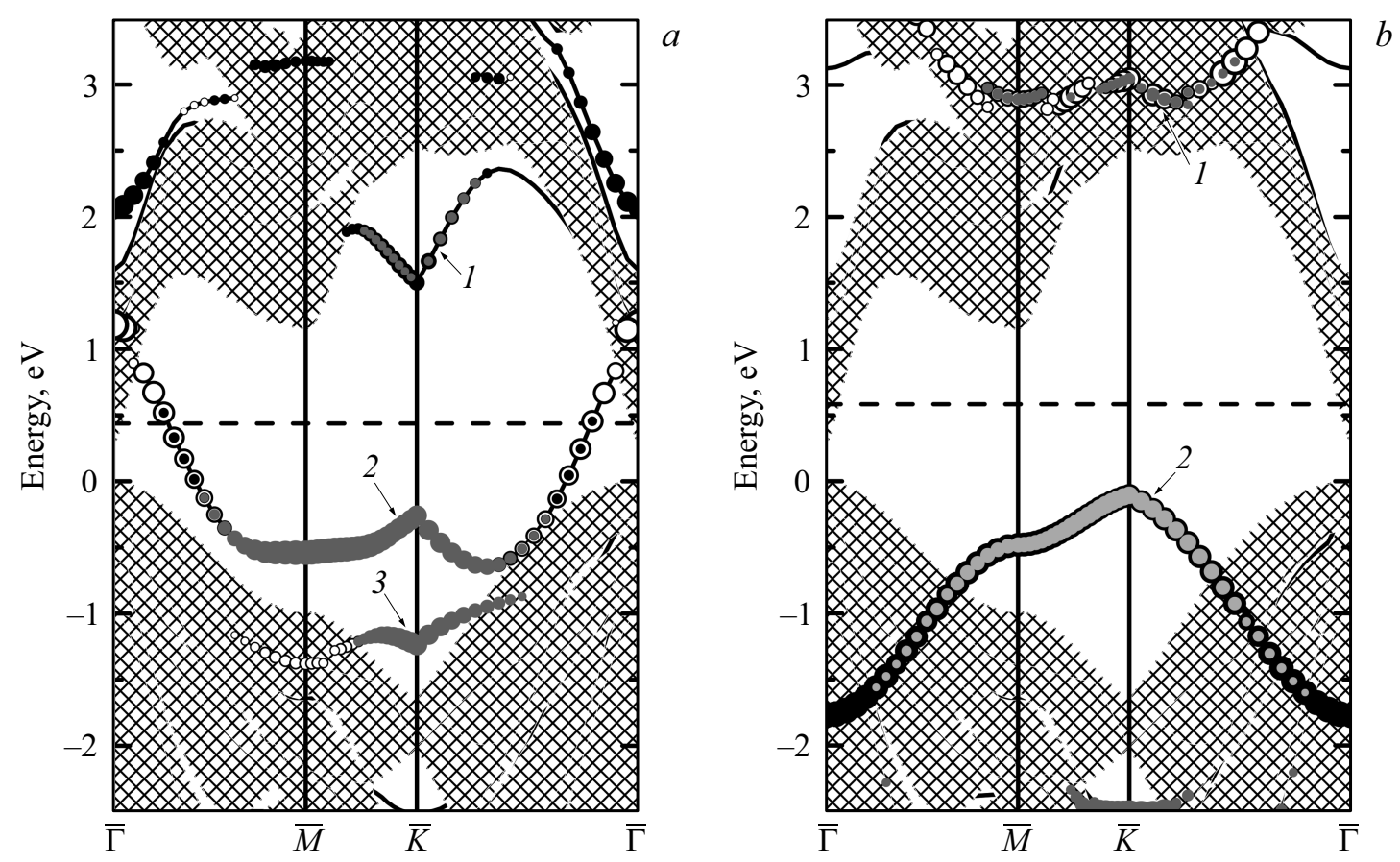

Рис. 7. Электронная структура поверхности $\operatorname{InSb}(111) \mathrm{A}-(1 \times 1)$ с адатомом кислорода и одним $(a)$ и тремя $(b)$ адатомами фтора.

Электронные энергетические спектры (рис. 6) при адсорбции фтора на поверхности демонстрируют меньшую плотность состояний в запрещенной щели, хотя структура этих состояний также сильно зависит от позиции адсорбции. Видно, что адсорбция фтора в наиболее предпочтительной In- $T$ позиции приводит к полному опустошению зоны, ответственной за пиннинг уровня Ферми на чистой поверхности (кривая 1 на рис. $3, a$ ), и к частичному опустошению состояний вблизи вершины валентной зоны в точке $\bar{\Gamma}$ (состояния 1 на рис. $6, a$ ). Состояния фтора при этом располагаются значительно ниже уровня Ферми, при энергиях $\sim-3.5$ эВ, и не приведены на рис. $6, a$. Аналогичное влияние фтора на электронную структуру поверхности наблюдалось и в случае полупроводников InAs и GaAs [25], однако состояния сурьмы располагались ниже вершины валентной зоны. Такое отличие, по-видимому, связано с бо́льшим переносом заряда от подповерхностного атома $\mathrm{Sb}_{2}(0.10 e)$ к фтору через гибридизацию с поверхностным атомом $\mathrm{In}_{1}$ по сравнению с переносом заряда от подповерхностных атомов $\mathrm{As}_{2}(0.01-0.03 e)$ на $\operatorname{InAs}(111) \mathrm{A}$ и $\mathrm{GaAs}(111) \mathrm{A}$ поверхностях. При адсорбции фтора в других позициях состояния, обусловленные $\operatorname{In}_{1}$ (кривая 1), также полностью опустошаются, а на $E_{\mathrm{F}}$ выходит зона, образованная преимущественно состояниями сурьмы (кривая 2), гибридизированными с $p$-состояниями фтора и $s, p$-состояниями индия (рис. $6, b-d$ ). Имеет место ее частичное опустошение вблизи $\bar{\Gamma}$-точки двумерной ЗБ в случае фтора в In- $B$ и $\mathrm{Sb}_{4}-T$ позициях, а также в окрестности точки $\bar{K}$, если фтор адсорбируется в $\mathrm{Sb}_{2}-T$ позиции.

\section{3. Коадсорбция кислорода и фтора}

При коадсорбции фтора и кислорода понижается плотность поверхностных состояний в фундаментальной щели, хотя один атом фтора не удаляет их полностью. В целом фтор смещает индуцированные кислородом поверхностные состояния в сторону дна зоны проводимости. В случае адсорбции фтора в In- $T$ позиции (рис. 7, $a$ ) эти поверхностные состояния, локализованные в основном на атомах индия, располагаются вблизи дна зоны проводимости в окрестности точки $\bar{K}$ двумерной ЗБ (кривая 1). Поверхностные состояния в центре зоны Бриллюэна (в точке $\bar{\Gamma}$ ) являются в основном состояниями сурьмы, тогда как состояния, локализованные на атомах кислорода, сконцентрированы на границе зоны Бриллюэна в окрестности точек $\bar{M}$ и $\bar{K}$ двумерной ЗБ (кривые 2 и 3). Напомним, что атом кислорода вследствие высокой химической активности не только образует сильную связь с поверхностным индием, но и взаимодействует с атомом сурьмы второго от поверхности слоя, в результате чего появляются смешанные $\mathrm{Sb}-\mathrm{O}$ состояния. Отметим также, что поверхностные состояния, локализованные на атомах фтора при коадсорбции в вершинной позиции (рис. 7, $a$ ), расположены значительно ниже $($ на $\sim 3.5$ эВ) вершины валентной зоны и не показаны на рисунке. Эти поверхностные состояния также локализованы преимущественно в точке $\bar{K}$ двумерной ЗБ. Заметим, что поверхностные состояния, индуцированные кислородом, полностью удаляются из запрещенной щели при коадсорбции трех атомов фтора (рис. $7, b)$; вблизи края фундаментальной щели остаются только поверхностные состояния, обусловленные In-F 

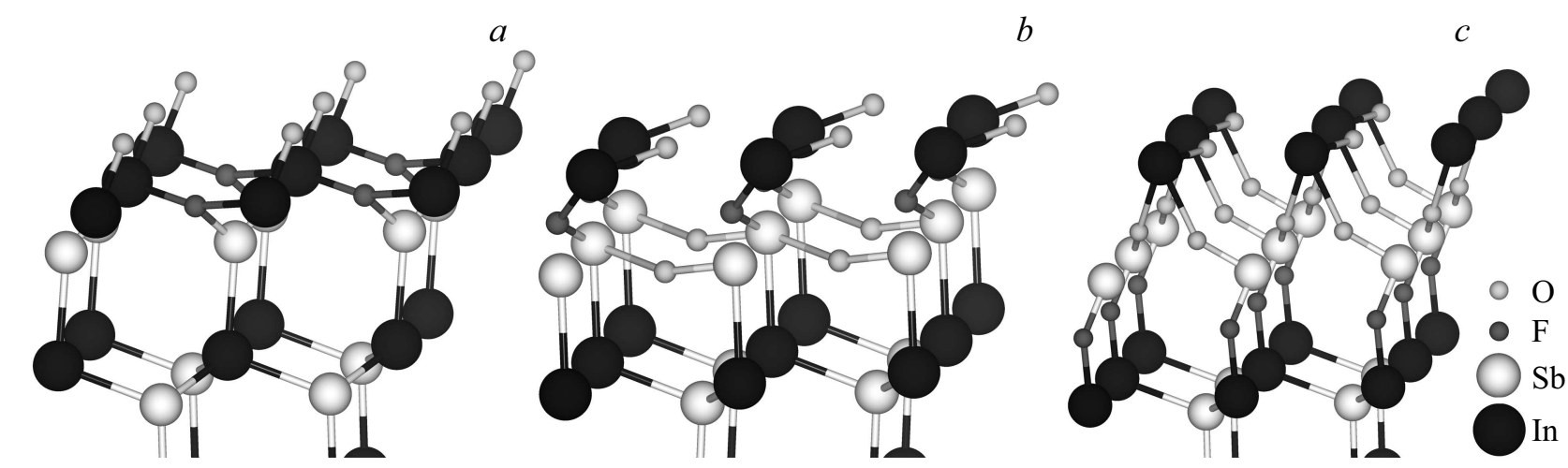

Рис. 8. Атомная структура поверхности $\operatorname{InSb}(111) \mathrm{A}-(1 \times 1)$ с кислородом, адсорбированным в мостиковой $B$-позиции, и одним $(a)$, двумя $(b)$ и тремя $(c)$ атомами фтора.

гибридизацией (кривая 1). Аналогичный эффект фтора имеет место и на поверхности (111)А полупроводников InAs и GaAs $[21,25]$. Однако в случае GaAs, который характеризуется широкой запрещенной щелью (1.42 эВ), кроме отмеченных выше состояний в щели оставались незанятые поверхностные состояния вблизи дна зоны проводимости вдоль направления $\bar{\Gamma}-\bar{K}$. На поверхности $\operatorname{InAs}(111) \mathrm{A}$, ширина запрещенной зоны которого значительно меньше (0.36 эВ), эти состояния располагались практически полностью в зоне проводимости. В настоящем расчете для узкозонного InSb (0.17 эВ), чья запрещенная зона в 2 раза уже, чем у InAs, незанятые поверхностные состояния полностью смещены в зону проводимости и располагаются на $\sim 0.5$ эВ выше дна этой зоны в точке $\bar{K}$ двумерной ЗБ.

Атомная структура поверхности $\operatorname{InSb}(111) \mathrm{A}-(1 \times 1)$, как было показано и в работе [25], значительно изменяется при коадсорбции двух электроотрицательных адсорбатов. Необходимо отметить, что длины связей атомов кислорода и фтора с атомами полупроводника при адсорбции в наиболее предпочтительных позициях равны $2.38 \AA\left(\mathrm{O}-\mathrm{In}_{1}\right), 2.00 \AA\left(\mathrm{O}-\mathrm{Sb}_{2}\right)$ и $2.01 \AA\left(\mathrm{F}-\mathrm{In}_{1}\right)$. Поскольку фтор, адсорбированный в вершинной позиции, смещает атом индия на $0.40 \AA$ по направлению к вакууму, по причине увеличения первого межслоевого расстояния атом кислорода может проникнуть в подповерхностную область. В результате атом кислорода находится на $0.37 \AA$ ниже поверхностного индия (рис. $8, a$ ). При коадсорбции двух атомов фтора наблюдается дальнейшее проникновение атомов кислорода, а также фтора в подповерхностные слои, где О располагается уже на $1.48 \AA$ А ниже атомов индия, в то время как атом фтора образует связи с сурьмой и находится на $0.35 \AA$ ниже второго от поверхности слоя сурьмы. При этом второй атом фтора остается на $0.53 \AA$ выше поверхностных атомов индия (рис. $8, b$ ).

В случае коадсорбции трех атомов фтора на ячейку (что соответствует увеличению концентрации фтора в окислительной среде) два из них, подобно атому кислорода, внедряются в подповерхностный слой, тогда как атом кислорода проникает еще глубже в подложку и локализуется между атомом сурьмы второго и атомом индия третьего от поверхности слоя (рис. 8,c). Это приводит к тому, что расстояние между атомами $\mathrm{Sb}_{2}-\mathrm{In}_{3}$ становится равным $3.77 \AA$, что превышает сумму их ковалентных радиусов. Таким образом, кислород разрушает связи в приповерхностных слоях и формирует новые $\mathrm{O}-\mathrm{Sb}_{2}$ и $\mathrm{O}-\mathrm{In}_{3}$ связи. Длины связей между атомами третьего-пятого атомных слоев практически не меняются, что указывает на локальный характер влияния адсорбатов на атомную структуру поверхности. Таким образом, увеличение концентрации фтора приводит к значительному изменению структуры подповерхностных слоев полупроводников вида $\mathrm{A}^{\mathrm{III}} \mathrm{B}^{\mathrm{V}}$, что связано с появлением фторсодержащего оксидного слоя, образующего ФАОС/ $\mathrm{A}^{\mathrm{III}} \mathrm{B}^{\mathrm{V}}$ с низкой плотностью поверхностных состояний. Как показали настоящие расчеты, атомная структура фторсодержащего оксидного слоя сильно зависит от концентрации фтора, а увеличение параметра решетки вследствие замещения мышьяка сурьмой способствует более легкому проникновению фтора в глубь полупроводника.

Отметим, что при моделировании влияния фтора кислород находился в наиболее выгодной мостиковой позиции, тогда как повышение концентрации фтора проводилось не последовательно, а одновременным размещением всех адатомов фтора над In-поверхностного слоя. В целом последовательность адсорбции кислорода и фтора, как показано в [26], несущественно влияет на структуру поверхностных состояний в запрещенной щели.

\section{4. Заключение}

Проведенные первопринципные расчеты атомной и электронной структуры поверхности $\operatorname{InSb}(111)$ с электроотрицательными адсорбатами показали, что независимо от типа поверхности наиболее предпочтительной позицией для адсорбции фтора является вершинная, а 
для кислорода - мостиковая позиция между поверхностными атомами. Энергия связи кислорода с поверхностью на 0.5-0.8 эВ больше, чем фтора. Адсорбция фтора на In-окончании поверхности $\operatorname{InSb}(111)$, как и в случае GaAs и InAs, приводит к устранению поверхностных состояний, образованных рz-орбиталями катионов, на нереконструированных поверхностях и откреплению уровня Ферми. В то же время адсорбция кислорода приводит к противоположным эффектам в зависимости от окончания поверхности $\operatorname{InSb}(111)$ : появляются дополнительные состояния в запрещенной щели на In-окончании и понижается плотность поверхностных состояний на Sb-окончании. Индуцированные кислородом поверхностные состояния полностью или частично удаляются из запрещенной щели при коадсорбции фтора в зависимости от его концентрации. Наблюдаются значительные структурные изменения в приповерхностных слоях полупроводника вследствие проникновения адсорбатов, что приводит к разрыву $\mathrm{In}-\mathrm{Sb}-$ связей и образованию новых $\mathrm{F}-\mathrm{Sb}$ - и $\mathrm{O}-\mathrm{Sb}$-связей. Последнее может рассматриваться как начальный этап образования фторсодержащего анодного оксидного слоя.

В целом установленные тенденции в изменении атомной и электронной структуры при адсорбции фтора и кислорода и их коадсорбции на поверхности $\operatorname{InSb}(111) \mathrm{A}$ согласуются с выявленными ранее на поверхностях полупроводников InAs и GaAs [26,26], что указывает на слабое влияние как анионов, так и катионов на взаимодействие электроотрицательных адсорбатов с поверхностью $\mathrm{A}^{\mathrm{III}} \mathrm{B}^{\mathrm{V}}(111) \mathrm{A}$. Полученные результаты способствуют более глубокому пониманию электронных свойств поверхности (111) полупроводников $\mathrm{A}^{\mathrm{III}} \mathrm{B}^{\mathrm{V}}$, что важно как для их практического применения, так и для прогнозирования электронных свойств тройных полупроводниковых соединений на их основе.

\section{Финансирование работы}

Работа выполнена в рамках проекта 23.2.8 ИФПМ СО РАН и программы повышения конкурентоспособности ТГУ. Численные расчеты выполнены на суперкомпьютеpe SKIF-Cyberia в Томском государственном университете.

\section{Конфликт интересов}

Авторы заявляют об отсутствии конфликта интересов.

\section{Список литературы}

[1] W. Mönch. Semiconductor Surfaces and Interfaces (Springer, Berlin, 1993).

[2] S.M. Sze. Physics of Semiconductor Device (Wiley Interscience Publication, N. Y., 1981).

[3] Ж.И. Алфёров. ФТП, 32, 3 (1998).

[4] Ж.И. Алфёров. УФН. Нобелевские лекции по физике, 172, 1068 (2002).
[5] D.L. Allara. Nature, 437, 638 (2005).

[6] C.L. Hinkle, E.M. Vogel, P.D. Ye, R.M. Wallace. Curr. Opin. Solid State Mater. Sci., 15, 188 (2011).

[7] N.N. Berchenko, Yu.V. Medvedev. Russ. Chem. Rev., 63, 623 (1994).

[8] Н.А. Корнюшкин, Н.А. Валишева, А.П. Ковчавцев, Г.Д. Курышев. ФТП, 30, 914 (1996).

[9] R.K. Ahrenkiel, L.L. Kazmenski, O. Jamjoum, P.E. Russel, P.J. Ireland, R.S. Wagner. Thin Sol. Films, 95, 327 (1982).

[10] G. Lucovsky, R.S. Bauer. J. Vac. Sci. Technol., 17, 946 (1980).

[11] N.A. Valisheva, O.E. Tereshchenko, I.P. Prosvirin, T.A. Levtsova, E.E. Rodjakina, A.V. Kovchavcev. Appl. Surf. Sci., 256, $5722(2010)$.

[12] N.A. Valisheva, M.S. Aksenov, V.A. Golyashov, T.A. Levtsova, A.P. Kovchavtsev, A.K. Gutakovskii, S.E. Khandarkhaeva, A.V. Kalinkin, I.P. Prosvirin, V.I. Bukhtiyarov, O.E. Tereshchenko. Appl. Phys. Lett., 105, 161610 (2014).

[13] M.S. Aksenov, A.Yu. Kokhanovskii, P.A. Polovodov, S.F. Devyatova, V.A. Golyashov, A.S. Kozhukhov, I.P. Prosvirin, S.E. Khandarkhaeva, A.K. Gutakovskii, N.A. Valisheva, O.E. Tereshchenko. Appl. Phys. Lett., 107, 173501 (2015).

[14] V.M. Bazovkin, N.A. Valisheva, A.A. Guzev, V.M. Efimov, A.P. Kovchavtsev, G.L. Kuryshev, I.I. Lee, A.S. Stroganov. Proc. SPIE, 5126, 118 (2012).

[15] K. Ahrenkiel, L.L. Kazmenski, P.J. Ireland, O. Jamjoum, P.E. Russell, D. Dunlavy, R.S. Wagner, S. Pattillo, T. Jervis. J. Vac. Sci. Technol., 21, 434 (1982).

[16] S. Miyamura, Y. Kasai, Y. Yamamura, T. Inokuma, K. Iiyama, S. Takamiya. Jpn. J. Appl. Phys., 42, 7244 (2003).

[17] M. Scarrozza, G. Pourtois, M. Houssa, M. Caymax, M. Meuris, M.M. Heyns, A. Stesmans. Surf. Sci., 603, 203 (2009).

[18] W. Wang, G. Lee, M. Huang, R.M. Wallace, K. Cho. Microelectron. Eng., 88, 3419 (2011).

[19] S.M. Lee, S.-H. Lee, M. Scheffler. Phys. Rev. B, 69, 125317 (2004).

[20] А.В. Бакулин, С.В. Еремеев, О.Е. Терещенко, С.Е. Кулькова. ФТП, 45, 23 (2011).

[21] С.В. Еремеев, Н.А. Валишева, О.Е. Терещенко, С.Е. Кулькова. ФТП, 46, 53 (2012).

[22] A.V. Bakulin, S.E. Kulkova, S.V. Eremeev, O.E. Tereshchenko. J. Phys. Chem. C, 118, 10097 (2014).

[23] A. Bakulin, S. Kulkova, O.E. Tereshchenko, A. Shaposhnikov, I. Smolin. IOP Conf. Series: Mater. Sci. Eng., 77, 012002 (2015).

[24] А.В. Бакулин, С.Е. Кулькова. ФТП, 50, 171 (2016).

[25] A.V. Bakulin, S.E. Kulkova, M.S. Aksenov, N.A. Valisheva. J. Phys. Chem. C, 120, 17491 (2016).

[26] N.A. Valisheva, A.V. Bakulin, M.S. Aksenov, S.E. Khandarkhaeva, S.E. Kulkova. J. Phys. Chem. C, 121, 20744 (2017).

[27] P.E. Blöchl. Phys. Rev. B, 50, 17953 (1994).

[28] G. Kresse, D. Joubert. Phys. Rev. B, 59, 1758 (1999).

[29] G. Kresse, J. Hafner. Phys. Rev. B, 49, 14251 (1994).

[30] G. Kresse, J. Furthmüller. Comp. Mat. Sci., 6, 15 (1996).

[31] J.P. Perdew, K. Burke, M. Ernzerhof. Phys. Rev. Lett., 77, 3865 (1996).

[32] S.M. Sze. Physics of Semiconductor Device (N.Y., Wiley Interscience Publication, 1981).

[33] K. Shiraishi. J. Phys. Soc. Jpn., 59, 3455 (1990).

[34] H.J. Monkhorst, J.D. Pack. Phys. Rev. B, 13, 5188 (1976). 
[35] J. Heyd, G.E. Scuseria, M. Ernzerhof. J. Chem. Phys., 118, 8207 (2003).

[36] A.V. Krukau, O.A. Vydrov, A.F. Izmaylov, G.E. Scuseria. J. Chem. Phys., 125, 224106 (2006).

[37] I. Vurgaftman, J.R. Meyer, L.R. Ram-Mohan. J. Appl. Phys., 89, 5815 (2001).

[38] W. Tang, E. Sanville, G. Henkelman. J. Phys.: Condens. Matter, 21, 084204 (2009)

[39] J. Mäkelä, Z.S. Jahanshah Rad, J.P. Lehtiö, M. Kuzmin, M.P.J. Punkkinen, P. Laukkanen, K. Kokko. Sci. Rep., 8, 14382 (2018).

Редактор Г.А. Оганесян

\section{Effect of oxygen and fluorine adsorption on the electronic structure of $\operatorname{lnSb}(111)$ surface}

A.A. Fuks ${ }^{1}$, A.V. Bakulin ${ }^{1,2}$, S.E. Kulkova ${ }^{1,2}$, N.A. Valisheva ${ }^{3}$, A.V. Postnikov ${ }^{4}$

${ }^{1}$ National Research Tomsk State University, 634050 Tomsk, Russia

${ }^{2}$ Institute of Strength Physics and Materials Science, Siberian Branch, Russian Academy of Sciences,

634055 Tomsk, Russia

${ }^{3}$ Institute of Semiconductor Physics, Siberian Branch,

Russian Academy of Sciences,

630090 Novosibirsk, Russia

${ }^{4}$ University of Lorraine,

F-57078 Metz, France

Abstract The energetics of oxygen and fluorine bonding on the $\operatorname{InSb}(111)$ surface depending on its termination was studied by the projector augmented-wave method. It is shown that, on the In-terminated surface, adsorption of fluorine, depending on its concentration, leads to partial or complete removal of surface states induced by oxygen adsorption from the forbidden gap. The penetration of both adsorbates into the subsurface layers leads to the breaking of $\mathrm{In}-\mathrm{Sb}$ bonds and the formation of chemical bonds of fluorine and oxygen with the near-surface atoms of the substrate that is the initial stage of the formation of fluorinecontaining anodic oxide layer. In case of the Sb-terminated $\operatorname{InSb}(111)$, the adsorption of oxygen contributes to a decrease of the density of surface states in the forbidden gap. The general trends in the change of the electronic structure of the (111) surface during fluorine and oxygen coadsorption in the series of $\mathrm{A}^{\mathrm{III}} \mathrm{B}^{\mathrm{V}}$ semiconductors are discussed. 